\title{
On the number of diamonds in the subgroup lattice of a finite abelian group
}

\author{
Dan Gregorian Fodor and Marius Tărnăuceanu
}

\begin{abstract}
The main goal of the current paper is to determine the total number of diamonds in the subgroup lattice of a finite abelian group. This counting problem is reduced to finite $p$-groups. Explicit formulas are obtained in some particular cases.
\end{abstract}

\section{Introduction}

The relation between the structure of a group and the structure of its lattice of subgroups constitutes an important domain of research in group theory. The topic has enjoyed a rapid development starting with the first half of the '20 century. Many classes of groups determined by different properties of partially ordered subsets of their subgroups (especially lattices of subgroups) have been identified. We refer to Suzuki's book [11], Schmidt's book [7] or the more recent book [12] by the second author for more information about this theory.

An important concept of subgroup lattice theory has been introduced by Schmidt [8] (see also [9]): given a lattice $L$, a group $G$ is said to be $L$-free if the subgroup lattice $L(G)$ has no sublattice isomorphic to $L$. Interesting results about $L$-free groups have been obtained for several particular lattices $L$, as the diamond lattice $M_{5}$ or the pentagon lattice $N_{5}$. We recall here only that a group is $M_{5}$-free if and only if it is locally cyclic and, in particular, a finite group is $M_{5}$-free if and only if it is cyclic. Notice also that the class of

Key Words: abelian group, subgroup lattice, diamond lattice, section, $L$-free group. 2010 Mathematics Subject Classification: Primary 20D30; Secondary 20D60, 20K27.

Received: 09.02.2015

Accepted: 02.03.2015 
$L$-free groups can be extended to the class of groups whose subgroup lattices contain a certain number of sublattices isomorphic to $L$ (see e.g. [15]).

Clearly, for a finite group $G$ the above concept leads to the natural problem of counting the sublattices of $L(G)$ that are isomorphic to a given lattice $L$. In the general case this problem is very difficult. This is the reason for which in the current paper we will treat only the particular case when $G$ is a finite abelian group and $L=M_{5}$. In other words, we will determine the number $d m(G)$ of all diamonds in the subgroup lattice of a finite abelian group $G$. Recall that a diamond in $L(G)$ (also called a diamond of $G$ ) is a sublattice of $L(G)$ which is isomorphic to $M_{5}$. It is easy to see that such a sublattice is completely determined by a triple $(A, B, C) \in L(G)^{3}$ satisfying $A \wedge B=B \wedge C=C \wedge A$ and $A \vee B=B \vee C=C \vee A$.

The paper is organized as follows. In Section 2 we show that the study can be reduced to $p$-groups and we develop a general method to find $d m(G)$ for finite abelian $p$-groups. Section 3 deals with the particular cases of elementary abelian $p$-groups and of abelian $p$-groups of rank 2 . The computation of $d m(G)$ is also exemplified for a finite rank 4 abelian 2-group. In the final section some conclusions and further research directions are indicated.

Most of our notation is standard and will usually not be repeated here. Basic definitions and results on lattices and groups can be found in $[3,4]$ and $[6,10]$, respectively.

\section{The key results}

Let $G$ be a finite abelian group and $L(G)$ be the subgroup lattice of $G$. It is well-known that $G$ can be written as a direct product of $p$-groups

$$
G=\prod_{i=1}^{k} G_{i},
$$

where $\left|G_{i}\right|=p_{i}^{\alpha_{i}}$, for all $i=1,2, \ldots, k$. Since the subgroups of a direct product of groups having coprime orders are also direct products (see Corollary of (4.19), [10], I), it follows that

$$
L(G) \cong \prod_{i=1}^{k} L\left(G_{i}\right) .
$$

The above lattice direct decomposition is often used in order to reduce many combinatorial problems on $L(G)$ to the subgroup lattices of finite $p$-groups (see e.g. $[2,13,14])$. This can be also applied to our problem.

First of all, let us assume that $k=2$. The following theorem shows the way in which $d m(G)$ depends on $d m\left(G_{1}\right)$ and $d m\left(G_{2}\right)$. 
Theorem 2.1. Let $G$ be a finite abelian group having a direct decomposition of type $G=G_{1} \times G_{2}$ with $\left|G_{i}\right|=p_{i}^{\alpha_{i}}, i=1,2$, and $p_{1}, p_{2}$ distinct primes. Then the number of diamonds in the subgroup lattice of $G$ satisfies

$$
d m(G)=d m\left(G_{1}\right)\left|L\left(G_{2}\right)\right|+d m\left(G_{2}\right)\left|L\left(G_{1}\right)\right|+6 d m\left(G_{1}\right) d m\left(G_{2}\right) .
$$

Proof. We easily infer that the diamonds of $G$ are of one of the following three types:

a) $(A \times H, B \times H, C \times H)$, where $(A, B, C)$ is a diamond of $G_{1}$ and $H \in$ $L\left(G_{2}\right)$;

b) $(H \times A, H \times B, H \times C)$, where $(A, B, C)$ is a diamond of $G_{2}$ and $H \in$ $L\left(G_{1}\right)$;

c) $\left(A \times A^{\prime}, B \times B^{\prime}, C \times C^{\prime}\right),\left(A \times A^{\prime}, B \times C^{\prime}, C \times B^{\prime}\right),\left(A \times B^{\prime}, B \times A^{\prime}, C \times C^{\prime}\right)$, $\left(A \times B^{\prime}, B \times C^{\prime}, C \times A^{\prime}\right),\left(A \times C^{\prime}, B \times A^{\prime}, C \times B^{\prime}\right),\left(A \times C^{\prime}, B \times B^{\prime}, C \times A^{\prime}\right)$, where $(A, B, C)$ is a diamond of $G_{1}$ and $\left(A^{\prime}, B^{\prime}, C^{\prime}\right)$ is a diamond of $G_{2}$.

Obviously, this leads to the desired equality (1).

Example. For the abelian group $G=\mathbb{Z}_{2}^{2} \times \mathbb{Z}_{3}^{2}$ we have

$$
\begin{aligned}
d m(G) & =d m\left(\mathbb{Z}_{2}^{2}\right)\left|L\left(\mathbb{Z}_{3}^{2}\right)\right|+d m\left(\mathbb{Z}_{3}^{2}\right)\left|L\left(\mathbb{Z}_{2}^{2}\right)\right|+6 d m\left(\mathbb{Z}_{2}^{2}\right) d m\left(\mathbb{Z}_{3}^{2}\right)= \\
& =1 \cdot 6+4 \cdot 5+6 \cdot 1 \cdot 4=50 .
\end{aligned}
$$

Even if we will not give an explicit formula, it is clear that the above result can be extended for an arbitrary $k \geq 2$, and consequently the computation of $d m(G)$ is reduced to the computation of $d m\left(G_{i}\right), i=1,2, \ldots, k$. So, in the following we will focus only on finite abelian $p$-groups.

In order to find $d m(G)$ for a finite abelian $p$-group $G$ we need some auxiliary results. Recall first that a section of $G$ is a quotient of a subgroup of $G$. More precisely, every section $S$ of $G$ is perfectly determined by a pair $(H, K) \in$ $L(G)^{2}$ such that $H \subseteq K$. We easily infer that $S$ is isomorphic to a subgroup, or, dually, to a quotient of $G$. The following lemma gives a way of counting the number of sections of a fixed type in $G$ (notice that it can be also used together with the Goursat's lemma to count the subgroups of $G$ ).

Lemma 2.2. Let $G$ be a finite abelian p-group and $S$ be a section of $G$. Then the number of sections of $G$ that are isomorphic to $S$ is

$$
n_{S}(G)=\sum_{T \leq G, T \cong S}|L(G / T)| .
$$


Moreover, these sections can be divided into $n$ classes, one for each subgroup $T_{n} \cong S$ of $G$, and there is a bijection between the sections $K / H$ of a class $m$ and the subgroups $R$ of $G / T_{m}$ such that if $K / H$ corresponds to $R$, then $H \cong R$ and $G / K \cong\left(G / T_{m}\right) / R$.

Proof. The proof is based on a famous result due to Baer (see Theorem 8.1.4 of [7] or Theorem 4.2 of [11]) which states that every finite abelian group is selfdual. In this way, if $\mathcal{A}_{G}$ is the set of subgroup-quotient group pairs $(A, G / A)$ of $G$, then one can define a bijection $\delta_{G}: \mathcal{A}_{G} \rightarrow \mathcal{A}_{G}, \delta_{G}((A, G / A))=\left(A^{\prime}, G / A^{\prime}\right)$, where $A \cong G / A^{\prime}$ and $A^{\prime} \cong G / A$.

We have to count all pairs $(H, K) \in L(G)^{2}$ such that $H \subseteq K$ and $K / H \cong$ $S$, or equivalently all pairs $(H, K / H) \in \mathcal{A}_{K}$ such that $K / H \cong S$. Since these pairs can be split into disjoint classes, one for every subgroup $K$ of $G$, and each preselected bijection $\delta_{K}$ is defined on the class $\mathcal{A}_{K}$ and preserves it, we infer that counting the pairs $(H, K / H) \in \mathcal{A}_{K}$ satisfying $K / H \cong S$ is the same as counting the pairs $\left(H^{\prime}, K / H^{\prime}\right) \in \mathcal{A}_{K}$ satisfying $H^{\prime} \cong S$. These can be obtained by counting for every $H^{\prime} \cong S$ the number of $K^{\prime}$ 's satisfying $H^{\prime} \subseteq K$. For a fixed $H^{\prime}$, the number of such $K^{\prime}$ 's is $\left|L\left(G / H^{\prime}\right)\right|$. Thus

$$
n_{S}(G)=\sum_{H^{\prime} \leq G, H^{\prime} \cong S}\left|L\left(G / H^{\prime}\right)\right|,
$$

which completes the proof.

Next we will focus on a particular class of diamonds in $L(G)$. A diamond ( $A, B, C$ ) of $G$ will be called a primary diamond if $A \wedge B=B \wedge C=C \wedge A=1$ and $A \vee B=B \vee C=C \vee A=G$. The structure of finite abelian $p$-groups having such a diamond is very restrictive.

Lemma 2.3. Let $G$ be a finite abelian p-group and $(A, B, C) \in L(G)^{3}$ be a primary diamond. Then $A \cong B \cong C \cong S$ and $G \cong S \times S$.

Proof. It follows immediately that $G=A \times B=B \times C=C \times A$. Then $A \cong G / C$ and $B \cong G / C$, and consequently $A \cong B$. Similarly, one obtains $B \cong C$, completing the proof.

An explicit formula for the number of primary diamonds of a finite abelian $p$-group $G$ of type $S \times S$ is indicated in the following lemma.

Lemma 2.4. Let $G \cong S \times S$ be a finite abelian p-group. Then the number of primary diamonds in $L(G)$ is

$$
\frac{|A u t(S \times S)|}{6|A u t(S)|} .
$$


Proof. Write $G \cong S_{1} \times S_{2}$ with $S_{1} \cong S_{2} \cong S$. We count first the pairs $(A, B) \in L(G)^{2}$ satisfying $A \wedge B=1$ and $A \vee B=G$. It is clear that every automorphism $f$ of $G$ determines such a pair $(A, B)$ given by $A=$ $f\left(S_{1}\right)$ and $B=f\left(S_{2}\right)$. Conversely, a pair $(A, B)$ corresponds to at least one automorphism of $G$. However, many automorphisms can map to the same such pair. More exactly, if two automorphisms $f_{1}$ and $f_{2}$ map to $(A, B)$, then the transformation between them is of type $\left(g_{1}, g_{2}\right) \in A u t(S)^{2}$. Therefore the number of pairs $(A, B) \in L(G)^{2}$ such that $A \wedge B=1$ and $A \vee B=G$ is

$$
\frac{|A u t(S \times S)|}{|A u t(S)|^{2}} .
$$

We still need to form triples $(A, B, C) \in L(G)^{3}$ with $A \wedge B=B \wedge C=$ $C \wedge A=1$ and $A \vee B=B \vee C=C \vee A=G$. Assume next that the pair $(A, B)$ is fixed. Then $G \cong A \times B$ and $A \cong B \cong S$. We easily infer that the number of $C$ 's completing $(A, B)$ to a primary diamond of $G$ is equal to the number of isomorphisms between $A$ and $B$, or, equivalently, to the number of automorphisms of $S$. Thus the number of the above triples is

$$
|A u t(S)| \frac{|A u t(S \times S)|}{|A u t(S)|^{2}}=\frac{|A u t(S \times S)|}{|A u t(S)|} .
$$

However, these triples are ordered and consequently $G$ has

$$
\frac{|A u t(S \times S)|}{6|\operatorname{Aut}(S)|}
$$

primary diamonds, as desired.

We are now able to establish our main principle of counting the diamonds of finite abelian $p$-groups.

Theorem 2.5. The number of diamonds in the subgroup lattice of a finite abelian p-group $G$ is

$$
d m(G)=\sum_{S} n_{S \times S}(G) \frac{|A u t(S \times S)|}{6|A u t(S)|},
$$

where $S$ runs over all types of sections $S$ of $G$ and the numbers $n_{S \times S}(G)$ are given by (2).

Proof. Let $(A, B, C)$ be a diamond of $G$. Obviously, by putting $H=A \wedge B=$ $B \wedge C=C \wedge A$ and $K=A \vee B=B \vee C=C \vee A$, one obtains a pair $(H, K)$ 
of subgroups of $G$ such that $H \subseteq K$, that is a section $S^{\prime}$ of $G$. Moreover, $(A, B, C)$ is primary in $S^{\prime}$ and so $S^{\prime}$ is of type $S \times S$ (where $A \cong B \cong C \cong S$ ) by Lemma 2.3. We infer that $d m(G)$ can be obtained by counting all primary diamonds in all sections of type $S \times S$ of $G$. Notice that the number of these sections can be computed by using Lemma 2.2 , while the number of primary diamonds in such a section follows by (3). Hence (4) holds.

Finally, we recall the well-known formula for the number of automorphisms of a finite abelian $p$-group (see e.g. $[1,5]$ ), which will be used in all particular cases in Section 3.

Theorem 2.6. Let $G=\prod_{i=1}^{n} \mathbb{Z}_{p^{\alpha_{i}}}$ be a finite abelian p-group, where $1 \leq$ $\alpha_{1} \leq \alpha_{2} \leq \ldots \leq \alpha_{n}$. Then

$$
|A u t(G)|=\prod_{i=1}^{n}\left(p^{a_{i}}-p^{i-1}\right) \prod_{u=1}^{n} p^{\alpha_{u}\left(n-a_{u}\right)} \prod_{v=1}^{n} p^{\left(\alpha_{v}-1\right)\left(n-b_{v}+1\right)},
$$

where

$$
a_{r}=\max \left\{s \mid \alpha_{s}=\alpha_{r}\right\} \text { and } b_{r}=\min \left\{s \mid \alpha_{s}=\alpha_{r}\right\}, r=1,2, \ldots, n .
$$

In particular, we have

$$
\left|A u t\left(\mathbb{Z}_{p}^{n}\right)\right|=p^{\frac{n(n-1)}{2}} \prod_{i=1}^{n}\left(p^{i}-1\right)
$$

\section{Counting diamonds for certain finite abelian $p$-groups}

In the following let $p$ be a prime, $n$ be a positive integer and $\mathbb{Z}_{p}^{n}$ be an elementary abelian $p$-group of rank $n$ (that is, a direct product of $n$ copies of $\mathbb{Z}_{p}$ ). We recall first the well-known formula that gives the number $a_{n, p}(i)$ of subgroups of order $p^{i}$ in $\mathbb{Z}_{p}^{n}$, namely

$$
a_{n, p}(i)=\frac{\left(p^{n}-1\right) \cdots(p-1)}{\left(p^{i}-1\right) \cdots(p-1)\left(p^{n-i}-1\right) \cdots(p-1)}, i=0,1, \ldots, n .
$$

Write $n=2 m+r$ with $r \in\{0,1\}$. Then the $S \times S$-sections of $\mathbb{Z}_{p}^{n}$ are of type $\mathbb{Z}_{p}^{2 i}, i=1,2, \ldots, m$. For every $i$, the subgroups of $\mathbb{Z}_{p}^{n}$ isomorphic to $\mathbb{Z}_{p}^{2 i}$ are in fact all subgroups of order $p^{2 i}$ of $\mathbb{Z}_{p}^{n}$, and these have the same quotient $\mathbb{Z}_{p}^{n-2 i}$. Thus

$$
n_{\mathbb{Z}_{p}^{2 i}}\left(\mathbb{Z}_{p}^{n}\right)=a_{n, p}(2 i)\left|L\left(\mathbb{Z}_{p}^{n-2 i}\right)\right|=a_{n, p}(2 i) \sum_{j=0}^{n-2 i} a_{n-2 i, p}(j)
$$


by Lemma 2.2. On the other hand, Theorem 2.6 shows that

$$
\frac{\left|A u t\left(\mathbb{Z}_{p}^{2 i}\right)\right|}{6\left|\operatorname{Aut}\left(\mathbb{Z}_{p}^{2} i\right)\right|}=\frac{1}{6} p^{\frac{i(3 i-1)}{2}} \prod_{k=i+1}^{2 i}\left(p^{k}-1\right) .
$$

Then (4) leads to the following result.

Theorem 3.1. The number of diamonds in the subgroup lattice of the finite elementary abelian p-group $\mathbb{Z}_{p}^{n}$ is given by

$$
d m\left(\mathbb{Z}_{p}^{n}\right)=\frac{1}{6} \sum_{i=1}^{\left[\frac{n}{2}\right]} p^{\frac{i(3 i-1)}{2}} a_{n, p}(2 i)\left(\sum_{j=0}^{n-2 i} a_{n-2 i, p}(j)\right) \prod_{k=i+1}^{2 i}\left(p^{k}-1\right) .
$$

We exemplify the equality (5) for $n=4$.

Example. We have

$$
d m\left(\mathbb{Z}_{p}^{4}\right)=\frac{p\left(p^{2}+p+1\right)\left(p^{4}-1\right)\left(p^{5}-p^{4}+p+3\right)}{6}
$$

and in particular

$$
d m\left(\mathbb{Z}_{2}^{4}\right)=735 .
$$

Next we will focus on computing the number of diamonds for finite rank 2 abelian $p$-groups, that is for groups of type $\mathbb{Z}_{p^{\alpha_{1}}} \times \mathbb{Z}_{p^{\alpha_{2}}}, 1 \leq \alpha_{1} \leq \alpha_{2}$. The $S \times S$-sections are in this case of the form $\mathbb{Z}_{p^{i}}^{2}=\mathbb{Z}_{p^{i}} \times \mathbb{Z}_{p^{i}}, i=1,2, \ldots, \alpha_{1}$. For every $i$, we can easily check that $\mathbb{Z}_{p^{\alpha_{1}}} \times \mathbb{Z}_{p^{\alpha_{2}}}$ has a unique subgroup isomorphic to $\mathbb{Z}_{p^{i}}^{2}$ and its quotient is of type $\mathbb{Z}_{p^{\alpha_{1}-i}} \times \mathbb{Z}_{p^{\alpha_{2}-i}}$. By Theorem 3.3 of [14], we infer that

$$
n_{\mathbb{Z}_{p^{i}}^{2}}\left(\mathbb{Z}_{p^{\alpha_{1}}} \times \mathbb{Z}_{p^{\alpha_{2}}}\right)=\left|L\left(\mathbb{Z}_{p^{\alpha_{1}-i}} \times \mathbb{Z}_{p^{\alpha_{2}-i}}\right)\right|=\frac{1}{(p-1)^{2}} f_{p}\left(\alpha_{1}-i, \alpha_{2}-i\right),
$$

where

$f_{p}\left(x_{1}, x_{2}\right)=\left(x_{2}-x_{1}+1\right) p^{x_{1}+2}-\left(x_{2}-x_{1}-1\right) p^{x_{1}+1}-\left(x_{1}+x_{2}+3\right) p+\left(x_{1}+x_{2}+1\right)$, for all $0 \leq x_{1} \leq \alpha_{1}$ and $0 \leq x_{2} \leq \alpha_{2}$. Moreover, we have

$$
\frac{\left|A u t\left(\mathbb{Z}_{p^{i}}^{2}\right)\right|}{6\left|\operatorname{Aut}\left(\mathbb{Z}_{p^{i}}\right)\right|}=\frac{1}{6} p^{3 i-2}\left(p^{2}-1\right)
$$

by Theorem 2.6. Hence the following result holds. 
Theorem 3.2. The number of diamonds in the subgroup lattice of the finite rank 2 abelian p-group $\mathbb{Z}_{p^{\alpha_{1}}} \times \mathbb{Z}_{p^{\alpha_{2}}}, 1 \leq \alpha_{1} \leq \alpha_{2}$, is given by

$$
d m\left(\mathbb{Z}_{p^{\alpha_{1}}} \times \mathbb{Z}_{p^{\alpha_{2}}}\right)=\frac{p+1}{6(p-1)} \sum_{i=1}^{\alpha_{1}} p^{3 i-2} f_{p}\left(\alpha_{1}-i, \alpha_{2}-i\right),
$$

where the quantities $f_{p}\left(x_{1}, x_{2}\right)$ are indicated above.

Obviously, by using a computer algebra program a precise expression of $d m\left(\mathbb{Z}_{p^{\alpha_{1}}} \times \mathbb{Z}_{p^{\alpha_{2}}}\right)$ can be obtained from (6). This counting is more facile in some particular cases.

Corollary 3.3. For every $n \in \mathbb{N}^{*}$, we have:
a) $d m\left(\mathbb{Z}_{p} \times \mathbb{Z}_{p^{n}}\right)=n\left(\begin{array}{c}p+1 \\ 3\end{array}\right)$;
b) $d m\left(\mathbb{Z}_{2^{n}} \times \mathbb{Z}_{2^{n}}\right)=\frac{1}{49}\left(3 \cdot 2^{3 n+2}-49 \cdot 2^{n}+14 n+37\right)$.

We also exemplify the equality (6) for $\alpha_{1}=2$ and $\alpha_{2}=3$.

Example. We have

$$
d m\left(\mathbb{Z}_{p^{2}} \times \mathbb{Z}_{p^{3}}\right)=\frac{p(p-1)(p+1)^{2}\left(p^{2}-p+2\right)}{3}
$$

and in particular

$$
d m\left(\mathbb{Z}_{4} \times \mathbb{Z}_{8}\right)=24 .
$$

In the end of this section we will apply Theorem 2.5 to count the diamonds of a rank 4 abelian 2-group, namely $G=\mathbb{Z}_{2} \times \mathbb{Z}_{4}^{3}$. These can be divided into four (disjoint) classes:

a) Those that are primary in $\mathbb{Z}_{2} \times \mathbb{Z}_{2}$-sections, 1 per section. We can easily see that $G$ has 35 subgroups isomorphic with $\mathbb{Z}_{2} \times \mathbb{Z}_{2}: 28$ have a quotient of type $\mathbb{Z}_{2} \times \mathbb{Z}_{4}^{2}$ and 7 have a quotient of type $\mathbb{Z}_{2}^{3} \times \mathbb{Z}_{4}$. Then Lemma 2.2 implies that the number of sections of type $\mathbb{Z}_{2} \times \mathbb{Z}_{2}$ in $G$ is

$$
n_{\mathbb{Z}_{2} \times \mathbb{Z}_{2}}(G)=28 \cdot\left|L\left(\mathbb{Z}_{2} \times \mathbb{Z}_{4}^{2}\right)\right|+7 \cdot\left|L\left(\mathbb{Z}_{2}^{3} \times \mathbb{Z}_{4}\right)\right|=28 \cdot 54+7 \cdot 118=2338,
$$

while the total number of primary diamonds in $\mathbb{Z}_{2} \times \mathbb{Z}_{2}$-sections is

$$
2338 \cdot 1=2338 .
$$


b) Those that are primary in $\mathbb{Z}_{4} \times \mathbb{Z}_{4}$-sections, $\frac{\left|A u t\left(\mathbb{Z}_{4} \times \mathbb{Z}_{4}\right)\right|}{6 \mid \text { Aut }\left(\mathbb{Z}_{4}\right) \mid}=8$ per section. $G$ has 112 subgroups isomorphic with $\mathbb{Z}_{4} \times \mathbb{Z}_{4}$, all of them having quotients of type $\mathbb{Z}_{2} \times \mathbb{Z}_{4}$. It follows that that the number of sections of type $\mathbb{Z}_{4} \times \mathbb{Z}_{4}$ in $G$ is

$$
n_{\mathbb{Z}_{4} \times \mathbb{Z}_{4}}(G)=112 \cdot\left|L\left(\mathbb{Z}_{2} \times \mathbb{Z}_{4}\right)\right|=112 \cdot 8=896,
$$

while the total number of primary diamonds in $\mathbb{Z}_{4} \times \mathbb{Z}_{4}$-sections is

$$
896 \cdot 8=7168
$$

c) Those that are primary in $\mathbb{Z}_{2}^{4}$-sections, $\frac{\left|A u t\left(\mathbb{Z}_{2}^{4}\right)\right|}{6\left|A u t\left(\mathbb{Z}_{2}^{2}\right)\right|}=560$ per section. $G$ has 1 subgroup isomorphic with $\mathbb{Z}_{2}^{4}$, whose quotient is isomorphic to $\mathbb{Z}_{2}^{3}$. It follows that the number of sections of type $\mathbb{Z}_{2}^{4}$ in $G$ is

$$
\left.n_{\mathbb{Z}_{2}^{4}}(G)=1 \cdot \mid L\left(\mathbb{Z}_{2}^{3}\right)\right) \mid=1 \cdot 16=16
$$

while the total number of primary diamonds in $\mathbb{Z}_{2}^{4}$-sections is

$$
16 \cdot 560=8960 \text {. }
$$

d) Those that are primary in $\mathbb{Z}_{2}^{2} \times \mathbb{Z}_{4}^{2}$-sections, $\frac{\left|A u t\left(\mathbb{Z}_{2}^{2} \times \mathbb{Z}_{4}^{2}\right)\right|}{6\left|A u t\left(\mathbb{Z}_{2} \times \mathbb{Z}_{4}\right)\right|}=3072$ per section. $G$ has 7 subgroups isomorphic with $\mathbb{Z}_{2}^{2} \times \mathbb{Z}_{4}^{2}$, all of them having quotients of type $\mathbb{Z}_{2}$. So, the number of sections of type $\mathbb{Z}_{2}^{2} \times \mathbb{Z}_{4}^{2}$ in $G$ is

$$
n_{\mathbb{Z}_{2}^{2} \times \mathbb{Z}_{4}^{2}}(G)=7 \cdot\left|L\left(\mathbb{Z}_{2}\right)\right|=7 \cdot 2=14,
$$

while the total number of primary diamonds in $\mathbb{Z}_{2}^{2} \times \mathbb{Z}_{4}^{2}$-sections is

$$
14 \cdot 3072=43008 \text {. }
$$

Hence

$$
d m\left(\mathbb{Z}_{2} \times \mathbb{Z}_{4}^{3}\right)=2338+7168+8960+43008=61474
$$

\section{Conclusions and further research}

All our previous results show that the problem of counting the number of sublattices in the subgroup lattice of a group $G$ that are isomorphic to a given lattice $L$ is an interesting computational aspect of subgroup lattice theory. Clearly, the study started in this paper can be extended for other lattices $L$ and groups $G$. This will surely be the subject of some further research. 
Finally, we indicate three open problems concerning the above topic.

Problem 5.1. Improve the results of Section 3, by obtaining an explicit formula for $d m(G)$ when $G$ is a finite abelian $p$-group of an arbitrary rank.

Problem 5.2. In the class of finite groups $G$ of a fixed order, find the minimum/maximum of $d m(G)$. Is it true that the function $d m$ is strictly decreasing on the set of types abelian $p$-groups of order $p^{n}$, totally ordered by the lexicographic order?

Problem 5.3. Determine the number of sublattices in other remarkable posets of subgroups (e.g. normal subgroup lattices) of a finite group that are isomorphic to a given lattice.

\section{References}

[1] Bidwell, J.N.S., Curran, M.J., McCaughan, D.J., Automorphisms of direct products of finite groups, Arch. Math. 86 (2006), 481-489.

[2] Birkhoff, G., Subgroups of abelian groups, Proc. Lond. Math. Soc. 38 (1934, 1935), 385-401.

[3] Birkhoff, G., Lattice theory, Amer. Math. Soc., Providence, R.I., 1967.

[4] Grätzer, G., General lattice theory, Academic Press, New York, 1978.

[5] Hillar, C.J., Rhea, D.L., Automorphisms of finite abelian groups, Amer. Math. Monthly 114 (2007), 917-923.

[6] Isaacs, I.M., Finite group theory, Amer. Math. Soc., Providence, R.I., 2008.

[7] Schmidt, R., Subgroup lattices of groups, de Gruyter Expositions in Mathematics 14, de Gruyter, Berlin, 1994.

[8] Schmidt, R., L-free groups, Illinois J. Math. 47 (2003), 515-528.

[9] Schmidt, R., L L 10 -free groups, J. Group Theory 10 (2007), 613-631.

[10] Suzuki, M., Group theory, I, II, Springer Verlag, Berlin, 1982, 1986.

[11] Suzuki, M., Structure of a group and the structure of its lattice of subgroups, Ergebnisse der Mathematik und ihrer Grenzgebiete, Neue Folge, Heft 10, Springer Verlag, Berlin-Göttingen-Heidelberg, 1956. 
[12] Tărnăuceanu, M., Groups determined by posets of subgroups, Ed. Matrix Rom, Bucureşti, 2006.

[13] Tărnăuceanu, M., A new method of proving some classical theorems of abelian groups, Southeast Asian Bull. Math. 31 (2007), 1191-1203.

[14] Tărnăuceanu, M., An arithmetic method of counting the subgroups of a finite abelian group, Bull. Math. Soc. Sci. Math. Roumanie (N.S.) 53/101 (2010), 373-386.

[15] Tărnăuceanu, M., A characterization of the quaternion group, Sci. An. Univ. "Ovidius" Constanţa 21 (2013), 209-214.

Dan Gregorian FODOR

Faculty of Mathematics

"Al.I. Cuza" University

Iaşi, Romania

E-mail: dan.ms.chaos@gmail.com

Marius TĂRNĂUCEANU

Faculty of Mathematics

"Al.I. Cuza" University

Iaşi, Romania

e-mail: tarnauc@uaic.ro 